\title{
La intersubjetividad como base epistémica de las disciplinas tecnocientíficas
}

\section{Intersubjectivity as an epistemic basis of technical-scientific disciplines}

\author{
Leonardo Garro Mena \\ Jenny Andrea Calderón Castro ${ }^{2}$ \\ Daniel Láscarez Smith ${ }^{3}$
}

\section{Resumen}

El presente artículo reflexiona sobre el valor epistémico y metodológico de la intersubjetividad social como base de toda pretensión de objetividad para los proyectos académicos-comunitarios con orientaciones tecnocientíficas o ingenieriles. A partir de una crítica a las típicas relaciones coloniales entre el mundo académico y el comunitario, se integra la idea de intersubjetividad social con el desarrollo tecnocientífico, concluyendo que la intersubjetividad puede ser utilizada como base en un proceso de diseño ingenieril modificado que parta del aprendizaje. La reflexión tiene como base la experiencia de dos años de trabajo interdisciplinario entre profesionales de las ciencias naturales y las ciencias sociales en comunidades rurales costeras, en las cuales se construyeron, mediante la investigación acción, tecnologías de tratamiento de aguas residuales.

Palabras claves: Desarrollo comunitario, Diseño ingenieril, Extensión universitaria, Ambiente, Construcción social del conocimiento

\begin{abstract}
This article reflects on the epistemic and methodological value of social intersubjectivity as the basis of any pretense of objectivity for academic-community projects with techno-scientific or engineering orientations. From a critique of the typical colonial relations between the academic world and the community, the idea of social intersubjectivity is integrated with the technological-scientific development, concluding that intersubjectivity can be used as a basis in a modified engineering design process that starts with a learning stage. The reflection is based on the experience of two years of interdisciplinary work between natural sciences and social sciences in coastal communities, in which wastewater treatment technologies were built through action research.

KeyWords: Community development, Engineering design, University extension, Environment, social construction of knowledge
\end{abstract}

1._Universidad de Costa Rica, Escuela de Ingeniería Química, leonardo.garromena@ucr.ac.cr 2._Universidad de Costa Rica, Escuela de Ingeniería Química, jenny.calderoncastro@ucr.ac.cr 3. Universidad Nacional de Costa Rica, Departamento de Física, daniel.lascarez.smith@una.cr

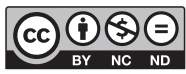




\section{Introducción}

Entre el año 2015 y 2017 se ejecutó un proyecto entre la Universidad Nacional, la Universidad de Costa Rica y el Laboratorio Nacional de Nanotecnología en las comunidades del distrito Lepanto, en el Golfo de Nicoya. El proyecto estuvo conformado por un equipo de trabajo de estudiantes y personal académico de ingeniería química, sociología, planificación económica, ciencias agropecuarias y gestión ambiental. El objetivo del proyecto fue diseñar e implementar, de forma participativa, soluciones para los problemas de residuos orgánicos y de aguas residuales que tanto han afectado a las personas en esta comunidad.

Ante esta situación surgieron algunas preguntas que orientaron las preocupaciones de carácter ontológico y epistemológico en un equipo tan diverso: ¿Cuál ha sido el tipo de abordaje metodológico y la concepción epistemológica de los proyectos académicos de extensión e investigación en las comunidades para la solución de problemas comunales? ¿Cómo se vinculan los conocimientos científicos entre disciplinas y estas a su vez con los conocimientos populares? ¿Cuál es el valor que se le otorga a los conocimientos populares para la solución de problemas aparentemente técnicos? ¿Cómo se hace un diagnóstico participativo cuando se trata de recolección de muestras de suelos y aguas? Al mismo tiempo surgían otras preguntas: ¿en qué consiste el aprendizaje dialógico y horizontal? ¿Cuáles son las condiciones para que estudiantes y académicos aprendan, escuchen y logren integrar nuevos conocimientos a los diseños?

El criterio técnico de las tecnociencias, las cuales, según Echeverría (2018) no tienen por guía el saber, como las ciencias, sino la innovación, normalmente está delimitado por una serie de referencias metodológicas y epistemológicas que indican la mejor forma o proceder de realizar algo. En el mundo de las relaciones universidad (ciencia, objetividad)-comunidades (saber popular, subjetividades) los problemas de carácter social o comunal son abordados por procedimientos que muchas veces impiden tener un "criterio técnico integral" para resolver problemas aparentemente técnicos como la construcción de infraestructura vial, para la recreación, para la comercialización, o para resolver, como en este caso, los problemas de aguas grises en comunidades, cuya afectación estaba caracterizada por los malos olores, por la restricción de espacios de esparcimiento, de salud pública para las personas y animales.

En este artículo se entiende la intersubjetividad como acto ético de la ciencia e irreductible a los métodos científicos. Se trata de un reconocimiento dinámico de las otredades en un contexto sociohistórico y cultural determinado. La intersubjetividad no es la suma de subjetividades personales, sino la existencia de mundos de la vida, es decir, intersubjetividades sociales en correlato con las condiciones objetivas de la sociedad, la cultura, la economía y la historia (Aristizábal, 2009). Así, por ejemplo, las familias que no tienen sistemas de drenaje de aguas negras y grises expresan condiciones materiales e históricas determinadas, pero las posibilidades intersubjetivas para explicarlo y solucionarlo implica un cierto tipo de acercamiento entre el mundo académico y comunal: implica reconocer al otro como sujeto histórico, se trata de un acto dialógico de conciencia sobre los conocimientos, las capacidades y el estatus social entre varias personas ubicadas en un proceso histórico, es decir, en un plano intersubjetivo altamente complejo.

Esta idea de lo intersubjetivo se distancia de los enfoques utilitaristas e instrumentalistas de la subjetividad como potencial para el valor agregado de una mercancía (estudios de percepciones y opiniones en el mercado, por ejemplo) y más bien sitúa el reto de la integración del plano intersubjetivo en la labor académica, caracterizada por la ausencia de esa consciencia. En esta reflexión se aborda la relación entre las tecnociencias e intersubjetividad como potencial epistemológico para la resolución de problemas comunales.

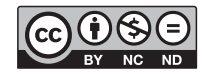


Esta discusión parte de los hechos históricos que limitan el potencial epistemológico de la intersubjetividad social en las ciencias sociales y naturales (pretensiones de objetividad, neutralidad, verificabilidad y subjetividad). Se toman los aportes generales del enfoque decolonial en la ciencia, el cual argumenta que la racionalidad científica está atravesada por un marco subjetivo colonial (no es objetivista como se afirma cotidianamente), que se expresa a través de categorías y conceptos científicos que reproducen desigualdades y subestiman el diálogo de saberes. Así, las disciplinas tecnocientíficas también legitimadas por el orden social, es decir, por los principios políticos-ideológicos de la modernidad capitalista, propusieron soluciones técnicas, ausentes de todo sentido histórico-político a los principales problemas sociales en las últimas décadas.

En la primera parte de este trabajo se desarrolla una crítica general a la relación entre modernidad y la valorización que la ciencia moderna hace de lo intersubjetivo como potencial transformador. La segunda parte especifica el caso de la ingeniería ambiental como disciplina tecnocientífica y su relación epistemológica con la intersubjetividad. La tercera parte presenta el proyecto junto con el abordaje metodológico y la estructura de trabajo, así como la relación entre el proceso de diseño, los aportes comunitarios y el aprendizaje de estudiantes, comunidad y cuerpo académico. La cuarta y última parte presenta algunos de los productos obtenidos gracias al abordaje ejecutado.

\section{Modernidad e intersubjetividad}

Existe una estrecha relación entre el proceso de constitución de la sociedad moderna y la ciencia. La crítica a la racionalidad moderna es una crítica de fondo a la conformación del mercado capitalista y a la ciencia como relatora de una sociedad moderna con presunciones científicas (Bautista, 2012) como base para el mantenimiento del orden social capitalista, eurocéntrico y patriarcal. El concepto de subalternización de lenguas y conocimientos utilizado por Garcés (2007) como proceso de clasificación ontológica de lo verdadero y universal desde los parámetros de la racionalidad moderna se expresa en las posibilidades de comunicación para establecer la horizontalidad en la otredad.

El lenguaje científico y el lenguaje popular han estado marcados por esos parámetros clasificatorios. Y como lo indican Castro-Gómez y Ramón Grosfogel (2007), la ciencia occidental, como conjunto de saberes y valores éticos, no puede separarse de su función ideología como legitimadora del orden imperante. Reconoce que la ubicación espacio- temporal en las relaciones coloniales explica en buena parte la negación y reconocimiento del otro.

En ese sentido, desde la lógica de una pretensión objetivista y de neutralidad, la ciencia moderna se basa en una visión de mundo construida subjetivamente desde los valores dominantes. Es subjetiva en tanto excluye otras visiones de mundo y otras racionalidades de las posibilidades de desarrollo alternativo. Las prácticas académicas, de nuestras universidades, no escapan a las relaciones de dominación sobre el mundo que se dan en dinámicas internas, pero desde lógicas globales.

Desde esta perspectiva, la visión de desarrollo que científicamente se reproduce desde las universidades en las comunidades está relacionada con la desvalorización de otras formas de conocimiento como los saberes populares. Así, los casos en que el académico o la académica consciente o insuficientemente trata de imponer una visión técnica y economicista del desarrollo sin asociarla históricamente a las visiones de mundo y necesidades particulares de quienes sufren el problema, es decir, sin incluirlas dentro de planos intersubjetivos, terminan siendo insostenibles en el mediano plazo. Sin embargo, se debe llamar la atención que los planos intersubjetivos pueden estar mediados por consideraciones coloniales. El reto consiste en cómo la intersubjetividad decolonial sea la base epistémica para las tecnociencias.

Entonces, la intersubjetividad, desde la crítica general de las relaciones coloniales de poder implica el proceso de desestructuración del pensamiento científico y cultural que legitiman y sostienen esas

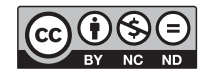

Rev. Reflexiones 98 (1) Enero-Junio, 2019: 61-74, E-ISSN: 1659-2859 
relaciones de poder. Eso, en el trabajo tecnocientífico, se traduce como la posibilidad de comunicación en el diálogo de saberes comunales, y que lo técnico es en realidad un tema político: ¿cómo y por qué se construyó una biojardinera de esta forma?

Según esta relación entre modernidad e intersubjetividad, el presente artículo considera que integrar la intersubjetividad como base epistémica en las disciplinas tecnocientíficas es un reto de carácter onto-epistemológico pues comienza por una reflexión crítica sobre los conceptos que se utilizan para analizar la realidad y un reto metodológico, pues implica reflexionar sobre las metodologías para la solución de los problemas comunitarios, sobre todo, en el marco académico de la extensión universitaria.

\section{La pretensión objetivista y la descontextualización histórica en la solución de problemas prácticos}

La objetividad ha sido una pretensión de gran utilidad en el contexto de los avances técnicocientíficos. Un punto de vista es más objetivo cuanto más independiente sea de la posición que ocupa el individuo en el mundo, así como de sus condiciones (Nagel, 1996). Por tanto, al objetivar observaciones se consigue desentrañar conclusiones de vasto ámbito de aplicación, como las leyes de la física. Esto genera un marco conceptual desde el cual es posible diseñar y construir objetos, productos, mercancías (tecnología), de manera determinista, así como predecir comportamientos, entre otras cosas. Gracias a la objetividad se pasa de la prueba y el error al diseño; o de la especulación a la predicción.

La objetividad como herramienta es, por tanto, no solo útil sino poderosa. Pero es quizá debido a su gran capacidad para generar conocimiento y avances técnico-científicos, que se ha convertido en una limitante para el desarrollo humano, cuando, por ejemplo, la objetividad "se vuelve al yo y trata de abarcar la subjetividad en la concepción de lo real" (Nagel, 1996, p. 14).

Según Nagel "la subjetividad de la consciencia es un aspecto irreductible de la realidad" (1996, p. 17), por tanto, la versión reduccionista del cientificismo no pasa de ser una visión parcial, incompleta e insuficiente que trata de aplicar teorías científicas para la comprensión de todo lo existente, como si estas no fueran un elemento más en el conjunto, sino un absoluto. Pero incluso, a pesar del reduccionismo cientificista, muchas veces resulta evidente la necesidad de inclusión de la subjetividad como parte de los procesos de diseño, como lo discuten Cho y Kim (2014) quienes presentan la aplicación de una metodología objetiva para la inclusión de elementos subjetivos dentro del diseño, o según queda patente en el análisis de León Duarte, Romero Dessens y Olea Miranda (2008), quienes estudian la inclusión de las necesidades tangibles e intangibles de las personas consumidoras (subjetividades) en el diseño industrial.

Otros estudios analizan este tema (Luo, Kannan, y Ratchford, 2008; D’Olivo, Curto, Faucheu, Lafon, Bassereau, Lê, y Delafosse, 2013), así como la integración de la subjetividad misma de quien se encarga de realizar el diseño (Sebastian, Ledoux, Collignan, y Pailhes, 2012). Sin embargo, dichos estudios se enmarcan casi de manera exclusiva en el diseño industrial, donde el efecto de la subjetividad es fácilmente identificable dados los hábitos de consumo. Por otra parte, el enfoque es integrar elementos subjetivos en el proceso de diseño, no replantear el proceso de diseño desde la subjetividad.

En la medida en que la objetividad es un convencionalismo ya es (inter)subjetiva, o sea, requiere de parámetros de aceptación de la comunidad científica, dicho conglomerado de expertos está compuesto por sujetos que convergen en creer en la validez de un postulado. Así pues, parece evidente que, en el caso específico del diseño industrial, la integración de elementos subjetivos es indispensable. No quiere esto decir que, la objetividad no tenga lugar, recuérdese que esta es una herramienta poderosa,

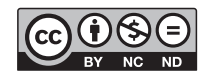

Rev. Reflexiones 98 (1) Enero-Junio, 2019: 61-74, E-ISSN: 1659-2859 
sino que, debido a las características del mercado de ciertos bienes de consumo, la subjetividad se torna indispensable. Y aquí cabe la pregunta ¿qué sucede con las otras disciplinas tecnocientíficas? ¿Acaso es el diseño industrial un caso único? Para responder a estas preguntas basta con comprender que los productos construidos con las bases técnico-científicas, en su gran mayoría, (si no totalidad) tienen por fin insertarse dentro de la sociedad para cumplir alguna función, por tanto, ¿cómo se podría ignorar el contexto social, la subjetividad, en el diseño?

Lo que sucede es que se ejerce la objetividad como un estandarte tiránico, que impone soluciones técnicas descontextualizadas a las necesidades específicas sin tomar en cuenta el efecto de las particularidades, de las subjetividades, y de las intersubjetividades históricas.

En las ciencias sociales, por otro lado, es común el discurso del reconocimiento de la otredad y de la subjetividad histórica (intersubjetividad) que supone ese reconocimiento. Pero sucede algo, epistemológicamente relevante, la incorporación de lo subjetivo en el plano social pasa por el filtro del conjunto instrumental del investigador que puede o no, facilitar el entendimiento del conjunto subjetivo del otro. Es así como las "consultas comunitarias", "audiencias públicas", la participación con "amplia representación social” pueden significar muy poco si no de definen las formas teórico- metodológicas para aprehender el contexto y en efecto, incorporar la visión de los "beneficiarios" del proyecto o de la política pública que se desea implementar. Esto se daría a partir de estrategias sectoriales que definan los rasgos intersubjetivos en el plano de lo social: la construcción de una autopista, de un puente o una política pública para pequeños exportadores agrícolas, muchas veces está basada en criterios de viabilidad que no reconocieron el principio intersubjetivo, sino, el de factibilidad financiera, por ejemplo.

\section{El caso de la Ingeniería Ambiental}

El proceso de diseño de proyectos en ingeniería, en general, consta de una serie de pasos, a saber: 1. Determinar las necesidades del cliente; 2. Establecer las especificaciones; 3. Desarrollo de conceptos, modelos e insumos; 4. Evaluación económica, optimización y selección; 5. Aprobación del cliente y construcción (Towler y Sinnott, 2013). Esta metodología se alinea con lo que Moriarty y Julliard (2001) describen como un mundo colonizado por la tecnología, en el que predominan la eficiencia y la productividad, en detrimento de la sociedad, causando, como describen Dym, Wesner, y Winner (2003), un abismo entre el mundo técnico y el humanista.

En lo que respecta a la Ingeniería Ambiental, donde se enmarca el proyecto base sobre el cual se desarrollaron las experiencias aquí descritas (desarrollo de alternativas de tratamiento de residuos orgánicos), el proceso de diseño se centra en la caracterización del efluente (variables físico-químicas), con base en la cual se desarrolla una alternativa de tratamiento que pueda ser instalada en el sitio, sin incluir de manera alguna aspectos sociales o de cualquier otra naturaleza. Recientes investigaciones en el diseño de sistemas de tratamiento (Molinos-Senante, Garrido-Baserba, Reif, Hernández-Sancho, y Poch, 2012) integran aspectos económicos e indicadores sociales aparte de las variables físico-químicas; sin embargo, incluso estas investigaciones se limitan a expandir las variables para tomar en cuenta aspectos de otras índoles, no a replantear el diseño en sí.

Por tanto, el proceso de diseño parece reproducir los vicios criticados por Quijano (2000) en su análisis de la colonialidad del poder, ya que el producto diseñado (los sistemas de tratamiento de desechos, en este caso), más que construirse se imponen, asumiendo que las únicas consideraciones dignas de tomarse en cuenta son las de quienes poseen conocimiento formal (académico), el cual, como ya se ha expuesto, en ocasiones no está contextualizado ni empoderado desde lo intersubjetivo.

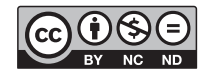

Rev. Reflexiones 98 (1) Enero-Junio, 2019: 61-74, E-ISSN: 1659-2859 
Desde otro punto de vista, pero bajo el mismo marco conceptual, los procesos de diseño y construcción de sistemas de tratamiento de desechos reproducen lo que Franz Fanon (1956) llamó "valor normativo", que no es más que una jerarquización cultural, la cual es definida de manera unilateral hegemónica, y establece que existen grupos humanos sin cultura, en nuestro caso, los procesos de diseño establecen, de manera implícita, que hay grupos humanos sin conocimientos dignos de tomarse en cuenta, aunque dichos grupos humanos sean quienes más conocimiento tienen sobre el lugar en el que viven, que es el contexto donde se instalará el sistema de tratamiento.

El reconocimiento de la otredad significa tener conciencia del valor social del otro en cuanto a su conocimiento, capacidad de análisis, preocupaciones y potencialidades para proponer soluciones. Implica también no personalizar el acto del reconocimiento, sino ubicarlo en un plano intersubjetivo. Este plano intersubjetivo puede ser conflictivo desde el punto de vista de los intereses comunales y las tensiones entre clases o grupos sociales, pero de ninguna manera le resta potencial a los diseños y ejecuciones en la planificación estratégica. El conflicto es parte del valor social y cultural de la subjetividad reconocida.

En este trabajo se plantea, como paso fundamental en el proceso de diseño, el aprendizaje, la inclusión y el respeto, sin jerarquías, de los saberes con que cuentan las personas involucradas. Con base en todos los saberes, y no sólo en los aportados por el grupo que está validado por la academia o la técnica, es que se propone el diseño y la ejecución de los proyectos.

\section{La intersubjetividad en el diseño ingenieril}

Con el objetivo de categorizar los procesos de diseño de sistemas de tratamiento de residuos, se proponen tres categorías, como se muestra en el Cuadro 1, dichas categorías dividen los procesos de diseño en tres niveles en función del grado de inclusión de la subjetividad. Los niveles 0 y 1 son los que ya se han discutido, en los que se realiza el diseño sin tomar en cuenta otros aspectos más que las variables físico-químicas (0) o agregando variables socio-económicas (1). Se propone un nuevo nivel (2), en el cual se replantea el proceso de diseño, se descolonializa al tomar los conocimientos formales (los utilizados por los niveles 0 y 1) y con ellos, por medio de una participación activa del grupo social para el cual se diseñará el sistema de tratamiento, se construye nuevo conocimiento, una amalgama entre los saberes técnico-científicos formales y los saberes del grupo social, proporcionados por su experiencia de vida.

Niveles del diseño de proyectos de acuerdo a su contextualización
Nivel
Elementos

$0 \quad$ Sólo considera aspectos técnicos. Sin contexto social.

1 Integra aspectos subjetivos en el diseño. Posee cierto contexto social sin repensar los fundamentos.

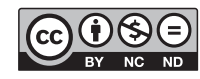


Nótese que no se trata de negar los conocimientos formales, ni de abandonar la objetividad, tales herramientas son indispensables, pero no suficientes. La propuesta se construye sobre la base de que la subjetividad de grupo social es irreductible, por tanto, los conocimientos técnico-científicos por sî mismos son insuficientes, se propone la construcción de un nuevo conocimiento, donde quienes tengan a cargo el desarrollo del proyecto expandan sus saberes por medio de los saberes de los otros, y de la construcción de nuevos contenidos teóricos, de nuevas categorías de análisis.

\section{El proyecto}

El anterior fundamento teórico fue aplicado en un proyecto de extensión realizado por un grupo de profesionales de diversas áreas (Ingeniería, Sociología, Planificación, Biología y Agronomía). El punto de partida del proyecto fueron dos comunidades rurales-costeras (Rosa Claus y Macondo) en el distrito de Lepanto, provincia de Puntarenas, Costa Rica.

El tema base del proyecto fue la disposición de residuos orgánicos, líquidos y sólidos, dada la ausencia en las comunidades en cuestión de sistemas centralizados de tratamiento e, incluso, de recolección en el caso de los residuos sólidos. En el planteamiento inicial se propuso como estructura base, en lo referente a la solución técnica, que los sistemas de tratamientos propuestos cumplieran con cuatro características esenciales: tratamiento, aprovechamiento, impacto ambiental y calidad de vida. El fundamento para tal estructura se presenta a continuación.

En la actual sociedad una estrategia para el tratamiento de residuos no puede ser sino integral, esto quiere decir que se encuentre sociocultural, política y económicamente definida, es por ello que, se establece el tratamiento como primer nivel de jerarquía, pues es la solución obvia y evidente a una problemática (la no adecuada disposición de los residuos). Se desprende del tratamiento, como implicaciones de su integralidad (o sea, su situacionalidad), el aprovechamiento y el impacto ambiental, y como consecuencia la calidad de vida.

El aprovechamiento de los residuos no sólo contribuye a la reducción de las implicaciones del siguiente concepto (impacto ambiental) sino que funge como fuerza motriz para el tratamiento. Al ser este (el tratamiento integral) socioculturalmente situado, será mejor aceptada una alternativa para el tratamiento que recompense de forma directa, visible e inmediata (al menos relativamente inmediata) a quienes la ejecuten, en comparación con una alternativa que solo ofrece recompensas popularmente abstractas como la mitigación del calentamiento global, la depuración atmosférica o la reducción en la contaminación oceánica.

La reducción del impacto ambiental no retribuye de forma visible los esfuerzos a micro escala, es por ello que el concepto de impacto ambiental sólo puede ser convertido en emprendimiento social por vía de la culturización o la retribución. Un escenario ideal sería potenciar el concepto anterior (aprovechamiento) en conjunto con una culturización en marcha para la elección de los métodos de tratamiento.

Finalmente, acciones como la realización de quemas locales pueden influir negativamente en la salud de la población, al utilizar un método alternativo para la disposición de materiales dichos efectos podrían mitigarse. El aprovechamiento, por otra parte, al brindar un beneficio tangible, podría colaborar en el aspecto socioeconómico. De esta forma, incluir la calidad de vida como elemento fundamental en la construcción de la estrategia para el tratamiento, no solo coloca a la persona como parte importante del esquema, y a su bienestar como activo que debe ser cuidado, sino que se contribuye a dinamizar la

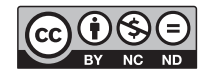

Rev. Reflexiones 98 (1) Enero-Junio, 2019: 61-74, E-ISSN: 1659-2859 
potencialización para el desarrollo y la permanencia del tratamiento y aprovechamiento de residuos, en el presente caso, específicamente, en los residuos orgánicos.

Lo anterior es, por supuesto, el punto de partida, la naturaleza del proyecto es la participacióncolaboración entre los diferentes sectores-grupos para construir soluciones con contexto, adaptadas y adecuadas a la situacionalidad que se les exige como productos que deben insertarse en la sociedad. Se busca trascender el proceso de diseño presentado por Towler y Sinnott (2013) y generar un nuevo paradigma en el que la base del diseño sea el aprendizaje. Por un lado, el aprendizaje por parte de las personas profesionales en tecnociencias, quienes se educarán gracias a los saberes de las personas que habitan el contexto donde se desarrollará el proyecto. Por otro lado, el aprendizaje de esas personas, en quienes se fomentará la apropiación de los conocimientos técnicos para que se integren al proceso de diseño. De esta manera, la subjetividad e intersubjetividad se tornan base epistémica sobre la que se construyen conocimientos, estos, por su parte, conforman la base sobre la que se lleva a cabo el diseño.

\section{Estructura de trabajo}

En la Figura 1 se presenta un esquema general de la estructura de trabajo, así como de las diversas interacciones entre los elementos que la componen. El punto de partida es una formulación inicial del proyecto, basada en lo descrito en el apartado anterior y en el conocimiento previo que se tenía de las comunidades. Tal punto de partida; sin embargo, y de acuerdo con la estructura misma de trabajo, es modificable conforme el proyecto avanza, conforme los conocimientos formales aportados por el equipo inicial se constituyen en conocimientos contextualizados gracias a la interacción y construcción que se realiza con las comunidades.

En síntesis, el diseño del proyecto somete la formulación inicial a la consideración de quienes participan en el proyecto, esto es: equipo formulador, estudiantes e integrantes de la comunidad. Esto se realiza por medio de reuniones (en el caso de estudiantes y equipo formulador) y visitas de campo (donde se integra a la comunidad). En las visitas de campo se realizan dos tipos de actividades: inspecciones en sitio, gracias a las cuales se añade el contexto físico y social, y reuniones-talleres con la comunidad, en las que se lleva una propuesta base y se busca el intercambio, la participación y la construcción de conocimiento conjunto, gracias al cual se rediseñan los productos para ser evaluados de nuevo a futuro. El proceso se repite cuantas veces sea necesario.

La forma en que las personas de las comunidades interactúan con el personal de la academia y el estudiantado ${ }^{1}$ refleja la forma en que las comunidades y sus propuestas pueden integrarse en el diseño. En una interacción unidireccional, como el proceso usual de diseño que se ha presentado previamente, la razón principal por la que se elige una alternativa de tratamiento por sobre las otras para una familia, es aquella que el personal académico a cargo del proyecto dicte, pues este tiene "autoridad intelectual". Sin embargo, si se parte del principio de no exclusión del conocimiento, el personal académico a cargo del proyecto llevará a cabo un proceso de aprendizaje, un diálogo cognitivo con la comunidad, y de este diálogo surgirá una razón entendida, justificada, aceptada por medio del consenso entre la diversidad de personas involucradas. Y si la propuesta es entendida, justificada y la solución construida en conjunto, entonces habrá empoderamiento, interacción e involucramiento.

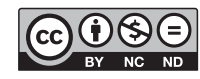


Figura 1

Estructura de trabajo

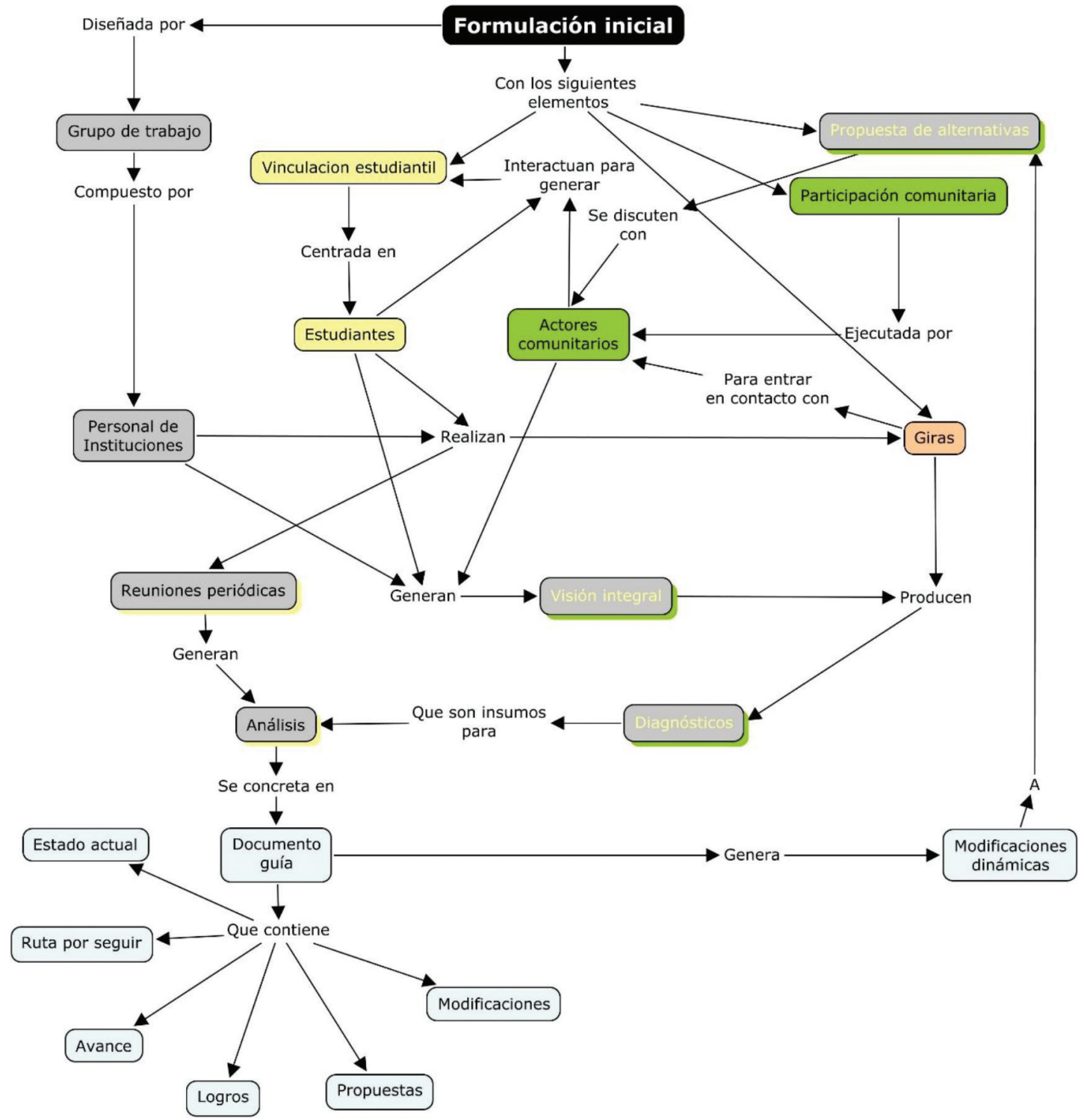

Fuente: Elaboración de las personas autoras.

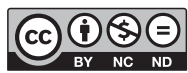

Rev. Reflexiones 98 (1) Enero-Junio, 2019: 61-74, E-ISSN: 1659-2859 
De esta manera, el objetivo del diseño se desplaza desde el objeto-producto, hasta la personausuaria. No se busca construir, por ejemplo, una biojardinera, sino de generar una alternativa al tratamiento de los residuos líquidos, de tal forma que dicha solución sea factible y perdurable, que las personas-usuarias se sientan hospitalarias ante objeto-producto (la biojardinera), que lo adopten no como un agente externo les invadió el espacio, sino como una construcción propia, doméstica, endógena, que se construyó, claro, en colaboración con personas que poseen otros saberes, pero que no se imponen, sino que dialogan.

\section{Resultados}

El proyecto, dada su naturaleza participativa, generó diversidad de productos tangibles. A continuación se presenta uno de ellos: un humedal artificial subsuperficial, o biojardinera, como se le conoce comúnmente (Figura 2). Las biojardineras son sistemas de tratamiento que aprovechan la capacidad de las plantas para absorber los nutrientes del agua (contaminantes) y purificarla.

En esta construcción en específico se llevó a cabo un aprendizaje diverso, tanto por parte de las personas que habitan el cuerpo generador de aguas residuales (una casa de habitación), quienes participaron desde etapas tempranas aportando información y tomando decisiones, hasta el estudiantado, con quienes se aprovechó para ilustrar conceptos teóricos en aplicaciones prácticas (como la caída de presión en lechos porosos, ilustrado por los diversos tamaños de piedra utilizados en la construcción). Pasando por situaciones como la adaptación de materiales, ya que en la zona no se contaba con todos los insumos necesarios y no se quiso recurrir a materiales que no estuvieran accesibles localmente, esto para garantizar la fácil reproducibilidad de las construcciones. A continuación, se profundizará en algunos de los detalles del proceso de diseño con el fin de evidenciar las interacciones y aprendizajes mencionados.

El aprendizaje como el primer paso del diseño: La premisa de la que se partió en este proyecto fue que el proceso de diseño usualmente utilizado en Ingeniería (tal como lo describe Towler y Sinnott, 2013) puede ser mejorado utilizando la subjetividad e intersubjetividad como base epistémica para la construcción de conocimiento en las disciplinas tecnocientíficas. Bajo esta premisa, las primeras etapas del diseño se dedicaron a aprender de la comunidad, sus necesidades, visión y expectativas. Esto resultó en una caracterización de la diversidad que permitió, por ejemplo, definir en dónde se instalarían biojardineras y dónde se aplicarían otras tecnologías.

Por otra parte, en el proceso mismo de construcción de las biojardineras se presentaron diversos problemas y situaciones que se resolvieron gracias al involucramiento de las personas de la comunidad logrado desde las primeras etapas. Por ejemplo, al ser zonas alejadas de la ciudad, muchas veces no encontramos los materiales adecuados para realizar la construcción; sin embargo, dado que las personas de la comunidad estaban involucradas en el proceso y comprendían los detalles técnicos, sugirieron alternativas con los materiales disponibles. Esto propició, en diversas ocasiones, la discusión entre la comunidad y el estudiantado, gracias a lo cual se propusieron soluciones alternas adaptadas al lugar y con los materiales locales.

Un ejemplo adicional consiste en las sugerencias de modificación en ciertos aspectos del diseño, como el método para colocar las piedras en la periferia de las biojardineras (Figura 2, a y b), ya que una de las personas de la comunidad sugirió cambios de tal manera que el resultado final tuviera la misma funcionalidad pero fuera superior en lo que respecta a la estética. Esto, desde el punto de vista de la Ingeniería, no suele tener valor alguno; sin embargo, cuando el producto final posee características más allá de su funcionalidad, como una estética agradable, este factor puede ser la diferencia entre el éxito

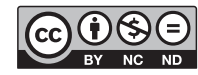

Rev. Reflexiones 98 (1) Enero-Junio, 2019: 61-74, E-ISSN: 1659-2859 
y el fracaso en el largo plazo, pues muchas veces las biojardineras son abandonadas por las personas usuarias debido a que no hay un vínculo con ellas, no se consideran un objeto que aporte valor.

La comunidad como fuente de conocimiento: El uso de mangueras de nivelusual entre las personas de las comunidades visitadas, esto es así pues muchas veces estas personas requieren realizar sus propias construcciones o colaboran en ellas para ahorrar dinero de mano de obra. Durante la construcción de la biojardinera se requirió el uso de este instrumento, lo cual una de las personas de la comunidad realizó sin ningún problema. Sin embargo, el estudiantado, no acostumbrado a realizar ese tipo de labores, mostró sorpresa ante el funcionamiento de dicho instrumento. El actor comunal les enseñó su uso y los detalles de su funcionamiento. Al final, una de las personas del cuerpo académico explicó al estudiantado que el nivel en ambos extremos de la manguera es el mismo pues y dado que la presión , la constante gravitacional y la densidad del agua son constantes, el nivel es el mismo.

Este ejemplo pareciera arbitrario, pues el instrumento en cuestión es muy simple, pero fue un hito en el proceso de aprendizaje del estudiantado ya que fueron conscientes de que sus conocimientos les permitían comprender el funcionamiento de la manguera de nivel, pero la persona de la comunidad sabía utilizarla, lo cual el estudiantado no pudo hacer sin ayuda. Al final la intervención del miembro del cuerpo académico realizó la conexión entre los conocimientos de la comunidad y los del estudiantado.

En síntesis, aprendimos que las personas usuarias, al adueñarse del producto desde las etapas tempranas de diseño, lo enriquecen con sus conocimientos, por ejemplo, al sugerir que se incorpore el paisajismo como parte de los criterios de diseño. En el caso de las biojardineras, éstas pasan a formar parte del hogar, pues se encuentran junto a la casa de habitación de la persona usuaria. Es lógico pensar que el diseño esté acorde con la estética particular que la persona prefiera, sin afectar, por supuesto, la funcionalidad.

Lo anterior nos hizo descubrir que el carácter intersubjetivo de algo a lo que usualmente no se le da importancia en ingeniería, como la apariencia, puede ser la clave del éxito o la causa del fracaso de un proyecto. Tiempo después, cuando se realizaron visitas de control, la biojardinera contaba con ciertas modificaciones, las cuales, aunque necesarias para el buen funcionamiento de esta, en el diseño eran imposibles de prever, pero fueron realizadas por la persona usuaria dado, en primer lugar, el conocimiento que había construido en el proceso, y en segundo lugar, la apropiación que hizo del producto, de tal manera que invirtió de su trabajo para mejorarlo, por iniciativa propia y sin necesidad de intervención externa.

En este caso, el proceso de diseño pasa de ser un monólogo, en el que se miden las variables fisicoquímicas del afluente, se realizan los cálculos y el dimensionamiento y al final se instala el producto, a ser un diálogo, en el cual el producto final es una construcción única, la cual, no sólo integra diversidad de criterios subjetivos e intersubjetivos, sino que genera aprendizajes en todas las partes involucradas, pues todas ellas cuentan con conocimientos, unos llamados formales, otros llamados informales, pero conocimientos que, en el contexto de este estudio. no tuvieron por qué jerarquizarse, sino sólo compartirse.

Nuestra valoración de la experiencia como profesionales es que el abordaje que realizamos le agrega un valor significativo al abordaje tecnocientífico tradicional. El seguimiento que hicimos de las tecnologías seleccionadas nos revelan que hay una alta sostenibilidad en el tiempo, las personas mantienen e incluso mejoran el funcionamiento de las biojardineras, agregando, por ejemplo, un techo para protegerla de las hojas (las cuales, si caen dentro de la misma y se descomponen, reducen su eficiencia) y reducir la incidencia de luz solar, lo cual retrasa el crecimiento y adaptación de las plantas en la etapa de adaptación.

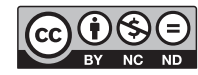


Las personas de la comunidad nos expresaron en repetidas ocasiones su interés por el proyecto, ya que en todo momento se sintieron incluidas en el mismo. Incluso se dio un incremento de participantes con el tiempo, ya que la misma comunidad comentaba de manera favorable el proyecto con comunidades o familias vecinas.

El estudiantado, por su parte, expresó que el principal valor encontrado en la experiencia fue el observar sus conocimientos teóricos puestos en práctica, muchas veces aprendiendo de las personas de la comunidad en conjunto con el cuerpo académico.

Como aspecto negativo resaltamos la no continuidad del proyecto, ya que al finalizar el mismo, aunque el aprendizaje y las experiencias se mantienen, la articulación lograda con las comunidades se rompe.

Figura 2

Etapas de la construcción del humedal artificial subsuperficial (biojardinera). (a) Etapa inicial, se coloca piedra cuarta, a través de la cual fluirá el agua, entrando en contacto con las raíces de las plantas. (b) Se completa el llenado con piedra. (c) Las plantas encargadas de consumir la carga orgánica son colocadas.

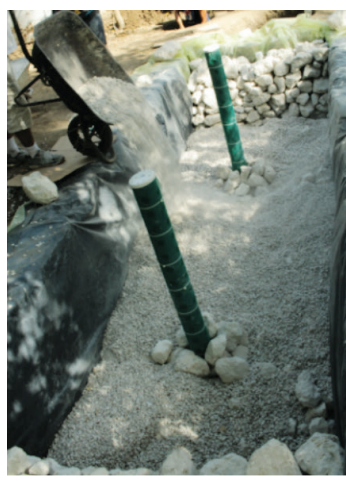

(a)

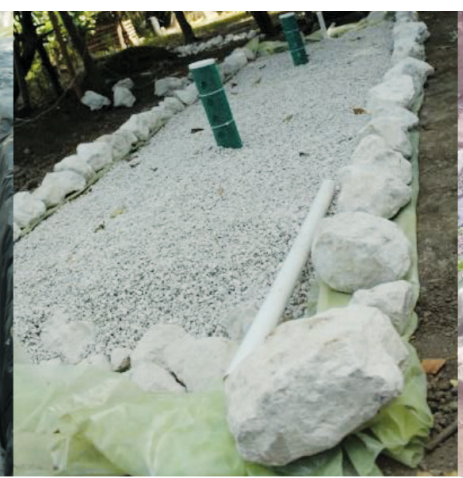

(b)

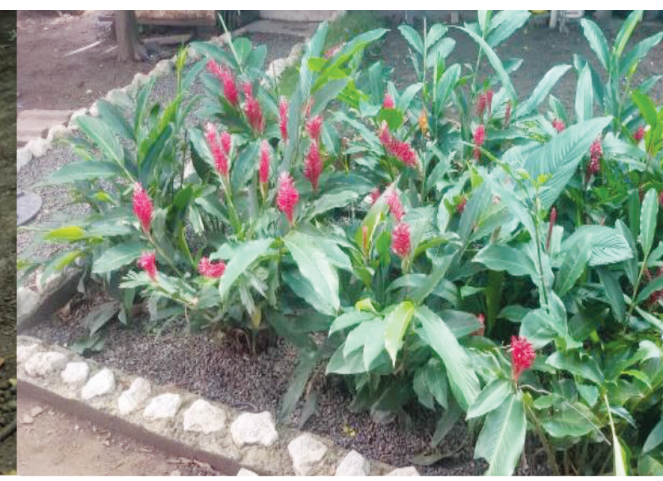

(c)

Fuente: Elaboración de las personas autoras.

\section{Conclusiones}

- Las propuestas de soluciones técnicas y tecnológicas para el desarrollo local comunitario que presentan las universidades, en el trabajo de acción social o extensión, deben enfocarse en incorporar las necesidades de las personas y de considerar en el diseño los conflictos sociales y los significados culturales de las prácticas cotidianas. Aún mejor, deben crear soluciones participativas y orientadas al éxito del proyecto en el tiempo a través de la idea de empoderamiento social.

- El cómo hacerlo significa una reconsideración epistemológica de cómo se construye el conocimiento; y una seria reconsideración de cómo las universidades incorporan al personal académico y al estudiantado en "el campo" o la "realidad". Es dejar la idea de laboratorios sociales, donde se ponen a prueba los conocimientos adquiridos en el aula, o se convierten las comunidades, en el espacio de construcción de nuevas relaciones sociales para el desarrollo a través de la aplicación creativa y critica de los conocimientos tecnocientíficos (ingenieriles) y los conocimientos locales- comunitarios.

- Al trabajar con los grupos de estudiantes se evidenció cómo el conocimiento técnico-científico se encuentra a veces aislado de sus equivalentes prácticos. Al trabajar con ciertas herramientas, como una manguera de nivel, las personas de la comunidad cuentan con el conocimiento sobre cómo

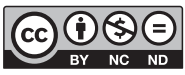


utilizarla, en tanto el grupo de estudiantes (en específico el perteneciente a Ingeniería Química) cuenta con el conocimiento sobre los principios hidráulicos de por qué funciona, pero a la hora de utilizarla no saben cómo porque no se ha hecho la relación entre teoría y práctica. Al interactuar se comparten conocimientos, y ambos, pobladores y estudiantes, resultan con experiencias que les expanden su concepción de las cosas.

- En el caso de la Ingeniería, el grupo de estudiantes constituye un grupo de profesionales a futuro, profesionales que se dedicarán a utilizar herramientas abstractas como los modelos y los datos para construir productos tangibles como los sistemas de tratamiento. Al realizar un proyecto participativo queda en evidencia que el producto de los modelos y datos es algo que debe integrarse a la sociedad, y por tanto, debe estar en función de esta. El diseño debe repensarse como un proceso cuyo primer paso es el aprendizaje de los conocimientos informales con que la sociedad cuenta, para, a partir de allí, avanzar en conjunto.

- Los científicos sociales que han trabajado en el proyecto han entendido que existen barreras cognitivas y culturales (propias y de los otros) que impiden una mediación del conocimiento en el trabajo de extensión. La revisión epistemológica constante de los presupuestos científicos y estereotipos culturales se trabajó en espacios de discusión y reflexión con observadores externos. Se realizaron también capacitaciones sobre inserción comunitaria, perspectiva de género, relaciones de poder, institucionalismo de los problemas y el objetivo de la extensión universitaria. Por ejemplo, luego de las sesiones de campo, se formaron grupos de reflexión sobre errores cometidos relacionados con la reproducción de roles de género entre el grupo de estudiantes, profesores y en la comunidad.

\section{Nota}

1. No se trata de simples etiquetas, sino que marcan pautas sociales. El personal académico se presenta a sí mismo como portador de conocimiento de mayor calidad o de alguna manera es percibido así por las personas de la comunidad.

\section{Referencias}

Aristizábal Hoyos Pedro Juan (2009). Intersubjetividad y comunicación. Universidad Tecnológica de Pereira Colombia. Acta fenomenológica latinoamericana. Volumen III (Actas del IV Coloquio Latinoamericano de Fenomenología) Círculo Latinoamericano de Fenomenología Lima, Pontificia Universidad Católica del Perú; Morelia (México), Universidad Michoacana de San Nicolás de Hidalgo 2009 - pp. 335-355.

Bautista Segales, Juan José. (2012). Hacia la descolonización de la ciencia social Latinoamericana. Cuatro ensayos metodológicos y epistemológicos. La Paz, Bolivia: Rincón ediciones.

Castro-Gómez, Santiago y Grosfoguel, Ramón -compiladores- (2007). El giro decolonial: reflexiones para una diversidad epistémica más allá del capitalismo global. Bogotá: Siglo del Hombre Editores; Universidad Central, Instituto de Estudios Sociales Contemporáneos y Pontifi cia Universidad Javeriana, Instituto Pensar.

Cho, Youngil y Kim, SuKyoung (2014). A Study on Affective Design by Subjective-Objective CoApproach. Vol. II. Hong Kong, 2014. http://www.iaeng.org/publication/IMECS2014/IMECS2014 _pp1120-1124.pdf

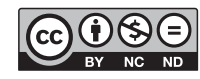

Rev. Reflexiones 98 (1) Enero-Junio, 2019: 61-74, E-ISSN: 1659-2859 
D’Olivo, Patrizia; Del Curto, Barbara; Faucheu, Jenny; Lafon, Dominique; Bassereau, Jean-François; Lê, Sebastian y Delafosse, David (2013). Sensory Metrology: when emotions and experiences contribute to Design (p. DS75-7_052). Presented at the 19th International Conference on Engineering Design (ICED13), The Design Society. Retrieved from http://hal-emse.ccsd.cnrs.fr/ emse-01063182/document

Dym, Clive L.; Wesner, John W. y Winner, Langdon. (2003). Social dimensions of engineering design: Observations from mudd design workshop III. Journal of Engineering Education, 92(1), 105-107. doi:10.1002/j.2168-9830.2003.tb00746.x

Echeverría, Javier (2018). De la filosofía de la ciencia a la filosofía de las tecno-ciencias e innovaciones. Revista iberoamericana de ciencia tecnología y sociedad 10.28 (2015): 105-14.

Fanon, Frantz (1956). Racismo y cultura. En Congreso de escritores y artistas negros. París.

Garcés Fernando (2007) Las políticas del conocimiento y la colonialidad lingüística y epistémica. El giro decolonial: reflexiones para una diversidad epistémica más allá del capitalismo global. Santiago Castro-Gómez y Ramón Grosfoguel -compiladores Bogotá: Siglo del Hombre Editores; Universidad Central, Instituto de Estudios Sociales Contemporáneos y Pontificia Universidad Javeriana, Instituto Pensar.

León Duarte, Jaime A.; Romero Dessens, Luis F. y Olea Miranda, Jaime (2008). Customer subjective perception as a main issue in conceptual product desing: A methodological proposal. Ingeniare. Revista Chilena De Ingeniería, 16(2), 301-309. doi:10.4067/S0718-33052008000200004

Luo, Lan; Kannan, P. K. y Ratchford, Brian T. (2008). Incorporating Subjective Characteristics in Product Design and Evaluations. Journal of Marketing Research, 45(2), 182-194.

Molinos-Senante, Maria, Garrido-Baserba, Manuel, Reif, R.; Hernández-Sancho, F. y Poch, M. (2012). Assessment of wastewater treatment plant design for small communities: Environmental and economic aspects. Science of The Total Environment. 427-428, 11-18. https://doi.org/10.1016/j. scitotenv.2012.04.023

Moriarty, G. y Julliard, Y. (2001). On subjectivity in focal engineering. Paper presented at the 177-184. doi:10.1109/ISTAS.2001.937736

Nagel, Thomas (1996). Una visión de ningún lugar. México: Fondo de Cultura Económica.

Quijano, Aníbal (2000). Colonialidad del poder y clasificación social. Journal of World-System Research, (2), 342-386.

Sebastian, Patrick; Ledoux, Y; Collignan, A y Pailhes, J. (2012). Linking objective and subjective modeling in engineering design through arc-elastic dominance. Expert Systems with Applications, 39(9), 7743-7756. https://doi.org/10.1016/j.eswa.2012.01.079

Towler, Gavin y Sinnott, Ray K. (2013). Chemical Engineering Design - Principles, Practice and Economics of Plant and Process Design (2nd Edition). Elsevier. Recuperado de: https://app.knovel. com/hotlink/toc/id:kpCEDPPEP4/chemical-engineering/chemical-engineering

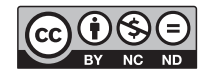

Rev. Reflexiones 98 (1) Enero-Junio, 2019: 61-74, E-ISSN: 1659-2859 